\title{
Efectos de la gestión y la calidad del agua potable en el consumo del agua embotellada
}

\author{
Effects of water quality and management on bottled water consumption \\ Alfonso Arellano $\mathbb{1}^{1 *}$, Verónica Lindao ${ }^{l}{ }^{l}$ \\ ${ }^{1}$ Facultad de Ingeniería, Universidad Nacional de Chimborazo, Riobamba, Ecuador, 060150; karilindao@ gmail.com \\ * Correspondencia: aarellano@unach.edu.ec
}

Recibido 11 agosto 2018; Aceptado 08 febrero 2019; Publicado 06 junio 2019

Resumen: Algunos autores han reportado el efecto de los factores demográfico, socio-económico y la calidad del agua, en el consumo de agua potable. Algunos estudios analizan el consumo de agua embotellada desde enfoques de salud pública y ecologista. Este artículo muestra las correlaciones entre la calidad del agua potable y la gestión realizada por las empresas administradoras; y, su efecto en el consumo de agua embotellada, en 11 ciudades. La gestión y la calidad del agua potable es evaluada por el Índice de gestión y de calidad del agua potable (Ingecap) que cuantifica el cumplimiento de: color, turbiedad, $\mathrm{pH}$, cloro residual, coliformes totales y sólidos totales disueltos (STD). La calidad del agua de la red pública es evaluada por las características organolépticas del agua (olor, color, sabor y presencia de tierra), reportadas por los usuarios. Se aplicaron: análisis de varianza, prueba de comparación de Tukey, tendencias lineales y coeficiente de determinación $\left(\mathrm{R}^{2}\right)$, apoyados en una estadística descriptiva. Los resultados de esta investigación confirman que la gestión y la calidad del agua potable inciden en el consumo de agua de la red pública, con marcadas diferencias en los estratos socio-económicos existentes en cada ciudad. El consumo de agua embotellada es el resultado de la desconfianza y de la calidad insatisfactoria del agua potable pública, percibida por el usuario. El consumo de bidones de agua depende de la capacidad económica del usuario. Se estableció una relación estadísticamente significativa entre las percepciones organolépticas de los usuarios y el Índice de Gestión y de Calidad del Agua Potable (Ingecap), que lo validaría como una herramienta fácil, barata y rápida para evaluar la gestión, administración y la calidad del agua potable para tomar correctivos inmediatos.

Palabras clave: Agua, calidad, embotellada, gestión, potable

Abstract: $\quad$ Some authors have reported the effects of demographic, socio-economic and water quality factors on drinking water consumption. Some studies report bottled water consumption from a public health and ecological perspective. This research shows the correlations between drinking water quality and management, and its effect on bottled water consumption, in 11 cities. The Ingecap Index quantifies the water quality and water management referring to standards of: colour, turbidity, $\mathrm{pH}$, residual chloride, total coliforms and total dissolved solids (TDS). The public water quality is also assessed by organoleptic characteristics such as: smell, colour, taste and soil presence. We evaluated the results through descriptive statistical methodology by using variance assessment, ukey comparison test, linear tendency and determination coefficient $\left(R^{2}\right)$. This research confirms the incidence of water quality on public drinking water consumption rates, with differences between economic and social levels. The consumption of bottled water is another effect of distrust in public drinking water quality. The users consume different amounts of bottled water according to their budget limitations. A significant statistical correlation was found between user's sensory perceptions and the Ingecap Index of management and drinking water quality. Ingecap suggests to be an easy, cheap and fast tool to assess water management as well as drinking water quality that contributes to make immediate improvements.

Keywords: Bottled, drinking, quality, management, water 


\section{Introducción}

Las preferencias de consumo de agua potable o embotellada varían de acuerdo a características demográficas y socio económicas (Ali Sajjadi, Alipour, Matlabi \& Biglari, 2015; Morote Seguido, 2017). La información obtenida en las investigaciones realizadas en las poblaciones Ecuatorianas entre el 2013 y 2015 muestran los patrones de los consumos de agua potable en rangos de poblaciones bien definidos (Arellano, Bayas, Meneses \& Castillo, 2018). Las ciudades pequeñas (menos de 8000 habitantes) son: Columbe (Sagñay \& Carguachi, 2015), Cubijíes (Samaniego \& Muela, 2015), Guamote, Chambo (Noriega, 2015), Químiag y Guano (Cáceres \& Rubio, 2015). Las ciudades medianas (entre 8000 y 30000 habitantes) son: La Joya de los Sachas (Barreno, 2015), Macas (Montenegro \& Tapia, 2014) y Guaranda (Patiño \& Pino, 2014). Las ciudades grandes (entre 30000 y 150000 habitantes) son Ventanas (Morillo \& Luna, 2013) y Riobamba (Carrillo \& Quintero, 2013). La información primaria contiene datos de: 1) la percepción de las características organolépticas del agua, 2) del consumo de agua embotellada en bidones, 3) del índice de gestión y calidad del agua potable (Ingecap); y 4) del consumo de agua de la red pública. La información 1 y 2 ha sido obtenida mensualmente a través de entrevistas con los residentes de los hogares "usuarios" en fichas previamente diseñadas. La información 3 se la registró mensualmente de los micro medidores de agua de la red pública que abastece a las residencias muestreadas, en cada ciudad durante un semestre. La información 4 ha sido obtenida de los reportes de los análisis físicos, químicos y microbiológicos realizados por los Municipios, Juntas Administradoras de Agua Potable o el Laboratorio de Servicios Ambientales de la Universidad Nacional de Chimborazo. El Ingecap (Carrillo \& Quintero, 2013) es un indicador cuantitativo representativo de las gestiones de control y de la calidad del agua de una ciudad. Este valor es expresado en porcentaje. Si el Ingecap es mayor que el $67 \%$ indica que el agua está en buenas condiciones. Cuando es menor que el $33 \%$ indica que su calidad y/o gestiones de control de calidad son deficientes. Un valor intermedio del Ingecap significa condiciones regulares. Para obtener este valor se ha considerado 6 parámetros de calidad de agua: (1) color, 2) turbiedad, 3) pH, 4) cloro residual, 5) coliformes totales y 6) sólidos totales disueltos (STD). Los valores de estos parámetros son frecuentemente reportados por los Municipios a través de las empresas administradoras de agua potable. Para calcular el
Ingecap se compara los valores de esos parámetros con la norma ecuatoriana INEN 1108 (Instituto Ecuatoriano de Normalización INEN, 2011), de calidad de agua potable y se califica su cumplimiento respecto a los rangos permitidos por dicha norma. Así mismo, se califica su incumplimiento en el caso de que no se hayan realizado las mediciones y pruebas correspondientes.

Se otorga un peso relativo a cada uno de los 6 parámetros, cuya suma total da 1 . Si no se han medido esos parámetros, el Ingecap mostrará una calificación baja porque no se ha realizado la gestión para medirlos, más no porque la calidad del agua sea deficiente. Los usuarios muestreados reportan percepciones de sabor, olor, color y existencia de tierra en el agua. El consumo de agua embotellada en bidones también ha sido registrado mensualmente. De estas variables, existe información proveniente de cada uno de 4 estratos socio-económicos definidos con el método de Arellano, González \& Gavilanes (2012). El factor económico ha sido reportado en varios estudios (Morote Seguido, 2017) por su efecto en el consumo de agua potable en varios países de diversos continentes. Se supone que podría existir una relación entre el consumo de agua embotellada con la calidad del agua de la red pública (INEC, 2016; Ali Sajjadi et al., 2015). Se espera que exista una relación directa entre el Ingecap y las percepciones organolépticas, que validarían el uso del Ingecap como indicador de las gestiones de control y de la calidad del agua potable (Rufener, Mäusezahl, Mosler \& Weingartner, 2010; Torres, Cruz \& Patiño, 2009). Estos parámetros serán llamados variables y serán asociadas a los consumos de los usuarios para determinar si la calidad del agua potable incide en la cantidad que consumen. Este estudio reporta el efecto de la gestión y de la calidad del agua potable pública, en el consumo de la misma y también en el consumo del agua embotellada, en diferentes estratos socio-económicos de 11 ciudades. No se considera el costo del agua embotellada porque fue constante durante la investigación y no es relevante en este análisis.

Los resultados se dividen en dos grupos. El primer grupo de resultados muestra la gestión y calidad del agua potable, expresadas a través de las percepciones organolépticas de los usuarios: olor, color, sabor y presencia de tierra, y el consumo del agua de la red pública, en cada uno de los 4 estratos socio económicos. El segundo grupo de resultados muestra la variación de consumos de agua embotellada, expresada en bidones per cápita, en las 11 ciudades. 
También muestra la correlación entre agua embotellada y la gestión y la calidad del agua de la red pública, cuantificada a través del Ingecap.

\section{Metodología}

El tamaño de las poblaciones de Químiag y Chambo (Arellano et al., 2018) han sido corregidas considerando las poblaciones del sector urbano, censadas en el 2010 (INEC, 2010). $\mathrm{Se}$ analizaron las relaciones de las variables en cada estrato socio económico en las 11 ciudades, dentro de los rangos poblacionales descritos arriba. El consumo mensual de bidones se registró para cada usuario (una sola vez en el semestre que duró la investigación en cada ciudad), y se dividió para el número de personas que habitaba en la residencia muestra correspondiente. Se usó la variable lt/hab-día de bidones de agua embotellada. Esta variable es calculada para estrato socio económico de una ciudad y es usado en este artículo con el nombre Bidones per cápita (lt/hab-día). La percepción de las características organolépticas es calculada como una fracción de usuarios de un estrato, que ha percibido cierta característica en el agua de la red pública. Esta variable será usada como porcentaje y corresponde a cada estrato socio económico. El Ingecap fue calculado mensualmente para algunas ciudades y para otras bimensualmente, de acuerdo a la información disponible por los Municipios o Juntas Administradoras de Agua Potable. El promedio aritmético de esos meses genera el Ingecap de una red de distribución de una ciudad en el semestre investigado. Si la ciudad tiene una sola red entonces el Ingecap es único en la ciudad. Si la ciudad tiene varias redes de distribución, el Ingecap será el promedio aritmético de todas las redes. La composición de los estratos socioeconómicos de una población, expresada en porcentaje, permite visualizar las diferencias entre cada ciudad, (figura 1) (Arellano et al., 2012). Esta estratificación es utilizada para calcular promedios ponderados de una variable representativa para una ciudad. Se lo expresa como una fracción porcentual de toda la ciudad. El estrato A es el de mayor capacidad económica y se le llama Alto. El estrato B es llamado medio alto. Al estrato $\mathrm{C}$ se lo llama medio Bajo y al estrato $\mathrm{D}$, que es de menor capacidad que los otros, se lo llama Bajo. Se han obtenido gráficos en donde se identifican tendencias visuales que son sometidas a análisis estadísticos descriptivos para ajustar un modelo lineal simple para estos conjuntos de datos. Los datos son lineales si el patrón gráfico de sus puntos se aproxima a una recta. Un modelo lineal viene expresado por una ecuación que contiene $m$ y $b$, estimados por el método de mínimos cuadrados.

$$
y=m x+b+\varepsilon
$$

Se calculó el coeficiente de determinación $\left(\mathrm{R}^{2}\right)$, que refleja la bondad del ajuste de un modelo de la variable que se pretende explicar. El resultado de $R^{2}$, oscila entre 0 y 1 . Cuanto más cerca de 1 se sitúe su valor, mayor será el ajuste del modelo a la variable que estamos intentando explicar. De forma inversa, cuanto más cerca de cero, menos ajustado estará el modelo y, por tanto, menos fiable será. Para considerar si una relación es significativa se consideró que el $\mathrm{R}^{2}$ debe ser mayor a 0.6. Cuando cumple este requisito se procedió a determinar el coeficiente de correlación R, según el criterio de Evans (tabla 1). Se considera una correlación valida entre 0.50 y 0.99 .

Tabla 1: Rango de correlaciones de Evans (Hernández Sampieri, Fernández Collado, \& Baptista Lucio, 2006).

\begin{tabular}{ll} 
Rango & \multicolumn{1}{c}{ Relación } \\
\hline 0.00 & No existe correlación \\
0.00 a 0.10 & Correlación débil \\
0.10 a 0.50 & Correlación media \\
0.50 a 0.75 & Correlación considerable \\
0.75 a 0.90 & Correlación muy fuerte \\
0.90 a 1 & Correlación perfecta \\
\hline
\end{tabular}

Las variables dependientes son: Bidones Per Cápita, Consumo per Cápita de agua potable de la red de distribución, y las percepciones organolépticas. Las variables independientes son: tamaño de la población y el Índice de la gestión y de la calidad del agua (Ingecap).

\section{Resultados y discusión}

De las 11 poblaciones en estudio (figura 2), el $63.64 \%$ posee una calificación de Ingecap regular mientras que tan solo un $36.36 \%$ tiene una calificación buena. Tres ciudades pequeñas (Guamote, Chambo y Guano) tienen una calificación de buena; y, 3 ciudades (Columbe, Cubijíes y Químiag) tienen regular. Una ciudad mediana (Guaranda) tiene la calificación de buena y 2 tienen regular (Joya de los sachas y Macas). Las 2 ciudades grandes (Ventanas y Riobamba) tienen un valor de Ingecap regular. 


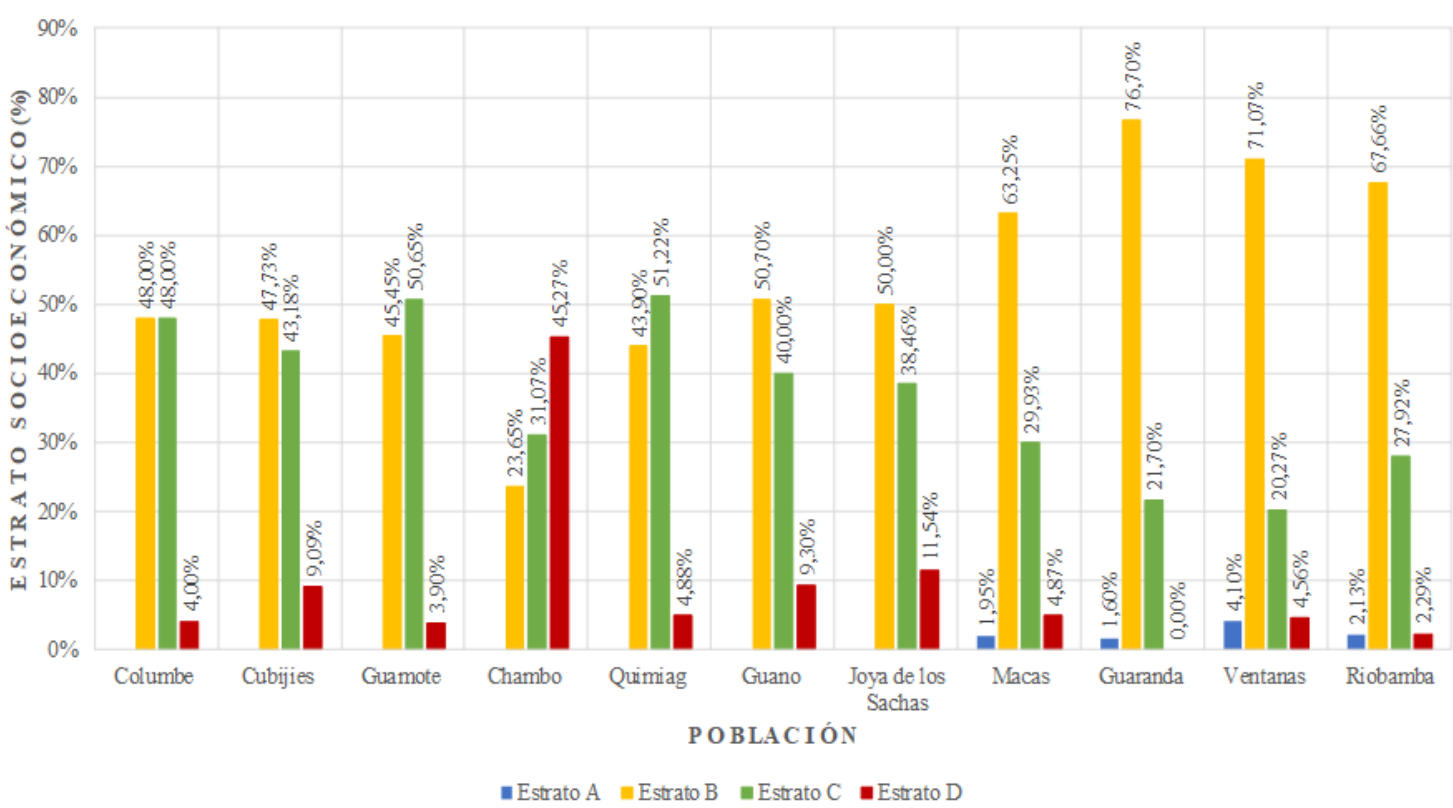

Figura 1: Distribución de los estratos socioeconómicos en las ciudades investigadas (Arellano et al., 2012).

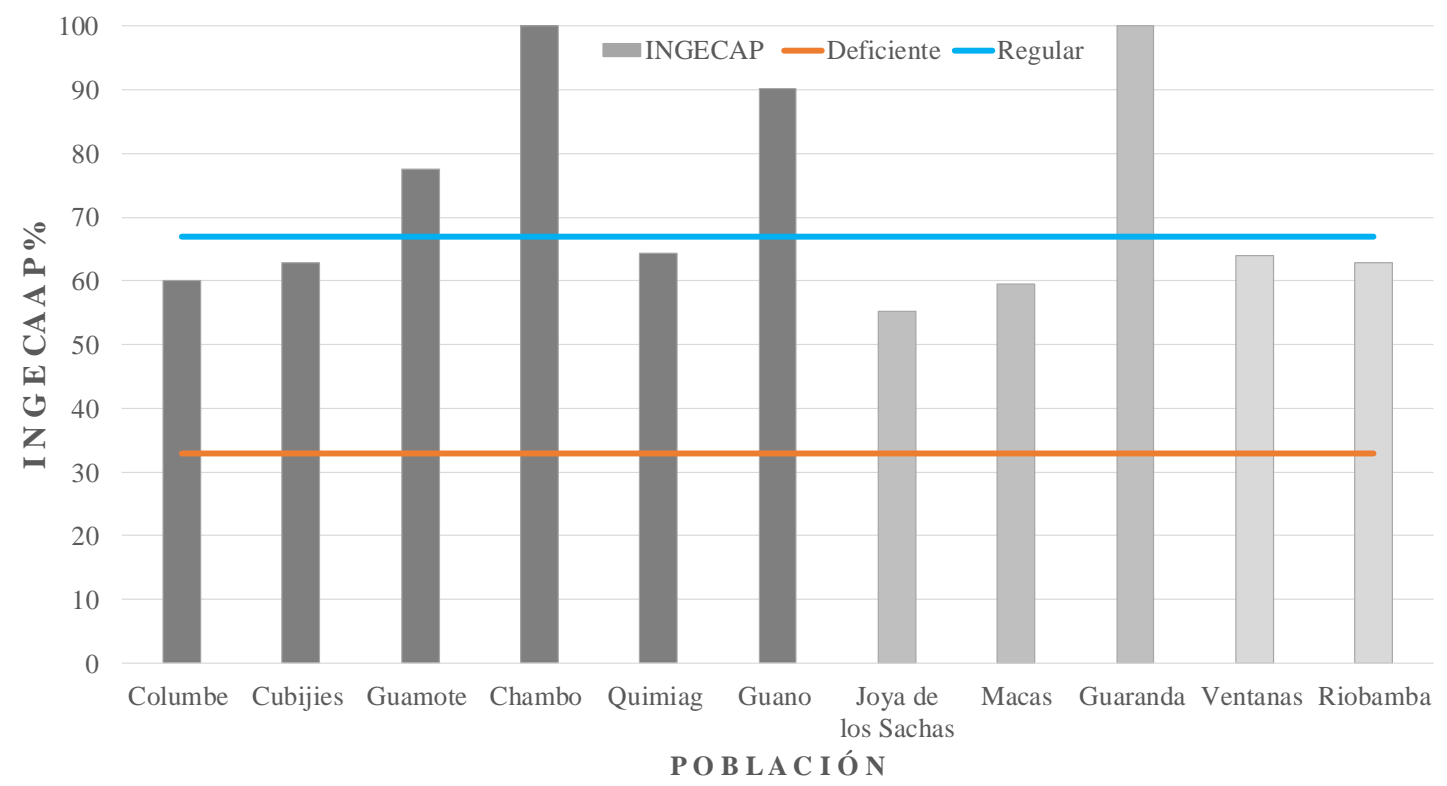

Figura 2: Ingecap en 11 ciudades Ecuatorianas (Lindao, 2018).

Aparentemente la gestión de la administración y la calidad del agua potable son mejores en las poblaciones pequeñas y decae cuando aumenta el tamaño de la población. Esto podría explicar la pendiente alta en las poblaciones pequeñas en el Modelo B-spline (Arellano, Bayas, Meneses \& Castillo, 2018). El consumo de agua en las poblaciones mayores de 500 habitantes sube vertiginosamente cuando sube la población, hasta alcanzar los consumos más altos alrededor de los 10000 habitantes. Cuando la población es mayor que los 10000 habitantes los consumos de agua potable bajan gradualmente.

Se evidencia en términos cuantitativos la correlación entre los factores económicos, demográficos y el consumo per cápita del agua de la red pública. Esto corrobora algunos estudios como los de (Morote Seguido, 2017; Ali Sajjadi et al., 2015). Sin embargo, ellos no han reportado la correlación entre el consumo, la calidad y la gestión del agua potable, como se lo ha cuantificado en este estudio. 
Tabla 2: Resultados del análisis estadístico del Ingecap vs Percepciones organolépticas (Lindao, 2018).

\begin{tabular}{clrrl} 
Estrato & Percepción & $\mathrm{R}^{2}$ & $\mathrm{R}$ & $\begin{array}{c}\text { Significancia } \\
(\mathrm{R})\end{array}$ \\
\hline \multirow{4}{*}{ A } & Olor & 0,9805 & 0,9902 & Co. Perfecta \\
& Color & 0,7894 & 0,8885 & Muy Fuerte \\
& Sabor & 0,6957 & 0,8341 & Muy Fuerte \\
& Tierra & 0,9788 & 0,9893 & Co. Perfecta \\
& Olor & 0,9819 & 0,9909 & Co. Perfecta \\
\multirow{4}{*}{ B } & Color & 0,8207 & 0,9059 & Co. Perfecta \\
& Sabor & 0,894 & 0,9455 & Co. Perfecta \\
& Tierra & 0,4725 & 0,6874 & Considerable \\
C & Olor & 0,7316 & 0,8553 & Muy Fuerte \\
& Color & 0,983 & 0,9915 & Co. Perfecta \\
& Sabor & 0,775 & 0,8803 & Muy Fuerte \\
& Tierra & 0,3638 & 0,6032 & Considerable \\
D & Olor & 0,8785 & 0,9373 & Co. Perfecta \\
& Color & 0,9939 & 0,9969 & Co. Perfecta \\
& Sabor & 0,6613 & 0,8132 & Muy Fuerte \\
& Tierra & 0,9953 & 0,9976 & Co. Perfecta \\
\cline { 2 - 5 } & \multicolumn{2}{c}{ Co. Perfecta: Correlación perfecta }
\end{tabular}

Los usuarios de la muestra de las 11 ciudades reportaron sus percepciones organolépticas del agua potable proveniente de la red pública (tabla 2.). Se podría suponer que al aumentar la calidad del agua potable las percepciones de olor, color, sabor y presencia de tierra, disminuyan. En los Estratos A y B existe una alta percepción de olor, color y sabor del agua potable, cuando el Ingecap es regular. Existe una correlación lineal positiva, perfecta o muy fuerte. Cuando el valor del Ingecap aumenta, las 4 percepciones disminuyen linealmente. Esto validaría el indicador Ingecap porque sus valores serían coherentes con las percepciones organolépticas de los usuarios.

En los estratos socio económicos C y D el color tiene un patrón diferente a los demás. El color aumenta cuando sube el Ingecap, lo cual parece contradictorio. Las percepciones de las otras 3 características: olor, sabor y presencia de tierra, tienen el mismo patrón que en los estratos A y B.

Estos resultados evidencian la coherencia entre los valores del Ingecap y las percepciones organolépticas por parte de los usuarios, en todos los estratos socio-económicos. Esto validaría al Ingecap como indicador de la gestión y de la calidad del agua potable.

Ahora se analiza otra variable que proporciona información del consumo de agua, que no proviene de la red pública (figura 3). La adquisición de bidones de agua potable, estaría relacionada con la insatisfacción y/o desconfianza del usuario con la calidad del agua de la red de distribución (Doria, 2006; Ali Sajjadi et al., 2015). Por esta razón se analiza los consumos per cápita de agua en bidones en los estratos socio-económicos de las 11 ciudades.

Tabla 3: Análisis de la Varianza del consumo de bidones per cápita de cada estrato de las 11 ciudades (Lindao, 2018).

\begin{tabular}{lrrrrr}
\multicolumn{1}{c}{ F.V. } & SC & gl & CM & F & p-valor \\
\hline Localidad & 0,87 & 10 & 0,09 & 2,98 & 0,0099 \\
Estratos & 0,40 & 3 & 0,13 & 4,58 & 0,0094 \\
Error & 0,87 & 30 & 0,03 & - & - \\
Total & 2,14 & 43 & - & - & - \\
\hline
\end{tabular}

$\mathrm{F} . \mathrm{V}=$ fuente de variación, $\mathrm{SC}=$ suma de cuadrados, $\mathrm{gl}=$ grados de libertad, $\mathrm{CM}=$ cuadrado menor, $\mathrm{FC}=$ Fisher calculado, $\mathrm{p}-$ valor $=$ probabilidad 


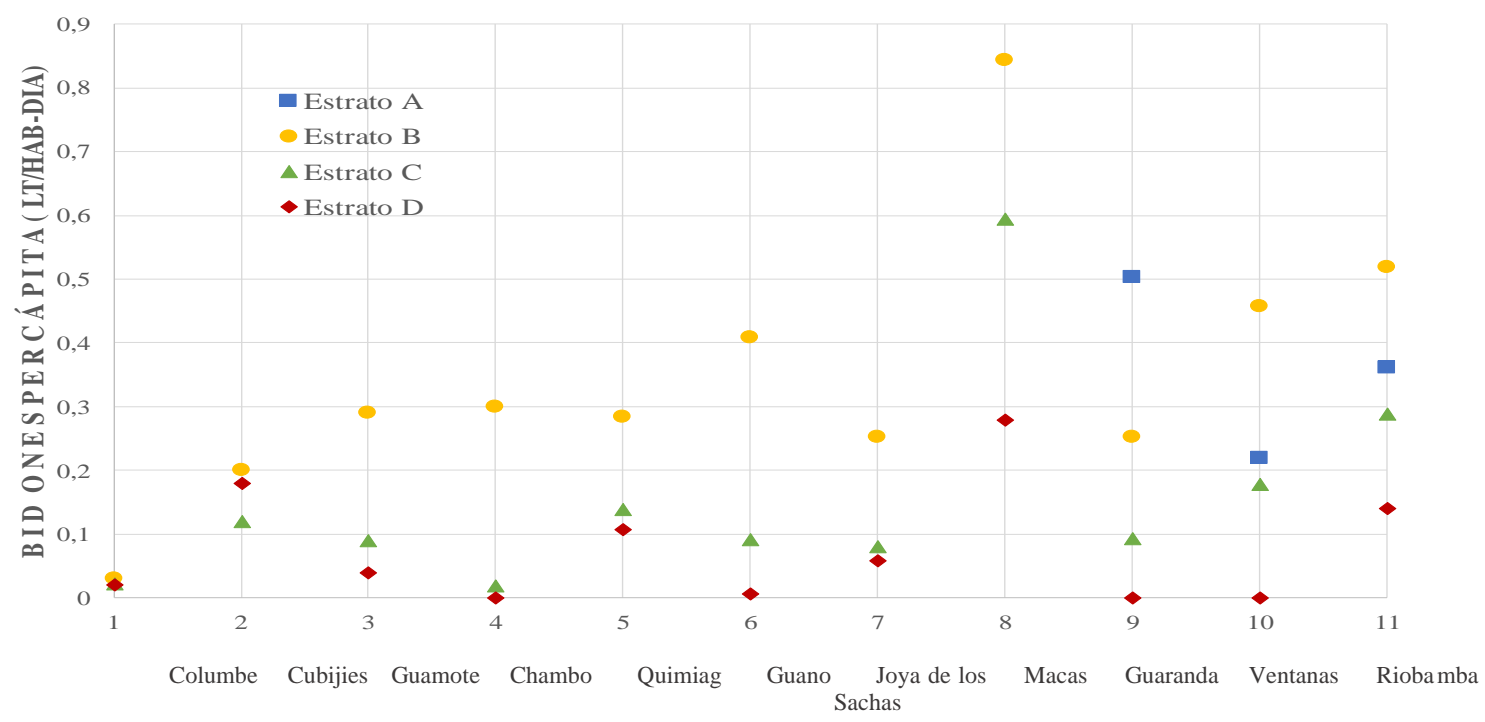

Figura 3: Consumo de agua embotellada en las 11 ciudades ecuatorianas (Lindao, 2018).

En el análisis de varianza para Bidones Per Cápita (tabla 3) existen diferencias altamente significativas para Localidades y Estratos. El consumo Per Cápita varía considerablemente en los factores estudiados.

La variación de consumo de bidones entre cada estrato socio económico, evidencia que el factor socio-económico siempre está presente en el consumo de agua. El estrato D, de menor capacidad económica (Arellano et al., 2012) consume menos que los otros estratos en todas las ciudades. El estrato socio económicos $\mathrm{B}$ es el más alto en las 7 ciudades en donde no existe el estrato A, consume más bidones per cápita en todas las ciudades con la excepción de Guaranda. Estos dos resultados demuestran que el consumo de agua embotellada les afectaría económicamente a los usuarios. Los usuarios que tienen mejor situación económica consumen más bidones de agua.

Tabla 4: Prueba de Tukey al 5\% para bidones per cápita (Lindao, 2018).

\begin{tabular}{lll}
\multicolumn{1}{c}{ Localidad } & Medias & Rango \\
\hline $\begin{array}{l}\text { Riobamba } \\
\text { Joya de los }\end{array}$ & 0,44 & $\mathrm{~A}$ \\
Sachas & 0,43 & $\mathrm{AB}$ \\
Ventanas & 0,39 & $\mathrm{AB}$ \\
Guaranda & 0,21 & $\mathrm{AB}$ \\
Macas & 0,21 & $\mathrm{AB}$ \\
Químiag & 0,13 & $\mathrm{AB}$ \\
Guano & 0,13 & $\mathrm{AB}$ \\
Cubijies & 0,13 & $\mathrm{AB}$ \\
Guamote & 0,11 & $\mathrm{AB}$ \\
Chambo & 0,08 & $\mathrm{AB}$ \\
Columbe & 0,02 & $\mathrm{~B}$ \\
\hline
\end{tabular}

Nota: Medias con una letra común no son significativamente diferentes $(\mathrm{p}>0,05)$

En la prueba de Tukey al 5\% (tabla 4) para Localidades se obtienen 3 rangos. Riobamba con un rango (A) con una media de 0.44 es la que más bidones Per Cápita consume. Columbe con un rango (B) y una media de 0.02 , es la que menos bidones Per cápita consume.

Tabla 5: Prueba de tukey al 5\% para consumo de bidones per cápita, por estratos (Lindao, 2018).

\begin{tabular}{ccl} 
Estratos & Medias & Rango \\
\hline B & 0,36 & A \\
A & 0,19 & A B \\
C & 0,17 & A B \\
D & 0,11 & B \\
\hline
\end{tabular}

Nota: Medias con una letra común no son significativamente diferentes $(\mathrm{p}>0,05)$

En la prueba de tukey al 5\% para Estratos (tabla 5), se obtienen 3 rangos. El estrato B con un rango (A) y con una media de 0.36 , es la que más bidones Per Cápita consume. El estrato D con un rango (B) y una media de 0.11 , es la que menos bidones Per Cápita consume. Los resultados de Tukey están resumidos en la tabla 6. Estos resultados evidencian la relación entre demografía, socioeconomía y consumo de agua embotellada y consecuentemente de agua potable, si se considera también los resultados analizados anteriormente.

En las ciudades grandes el consumo per cápita de bidones es mayor porque la desconfianza en la calidad del agua es alta debido a que la calidad del agua de la red es regular de acuerdo al valor del Ingecap (figura 2). 
Tabla 6: Análisis de Varianza para Bidones per cápita. Prueba de Tukey al 5\% para Localidades y Estratos (Lindao, 2018).

\begin{tabular}{cccccccc} 
Aspectos & p-valor & & Media & Rango & & Media & Rango \\
\hline Localidad & 0.0099 & Riobamba & 0.44 & A & Columbe & 0.02 & B \\
Estratos & 0.0094 & B & 0.36 & A & D & 0.11 & B \\
\hline
\end{tabular}

Se puede afirmar también que el consumo de agua embotellada es mayor cuando mayor es la capacidad económica del usuario. Además, se puede generalizar afirmando que el consumo de agua embotellada es mayor en las ciudades grandes debido a que existe mayor capacidad económica que en las ciudades medianas y pequeñas, lo cual se evidencia por la presencia del estrato A en las 4 ciudades más grandes mientras que en las otras ciudades solo existen estratos B, C y D (figura 1). Podría existir usuarios que desconfían de la calidad del agua de la red pública pero su economía no les permite adquirir el agua embotellada. Al obtener resultados significativos de consumo de bidones se podría afianzar los estudios realizados por (Doria, 2006) en los que afirma que el aumento en el consumo de agua embotellada se debe, entre otras cosas, a la desconfianza en las redes de suministro, o a las probables evidencias de que su calidad no sea la adecuada.
Ahora se analiza la relación entre el Ingecap y el consumo per cápita de bidones de agua, en cada estrato socio económico, de las 11 ciudades (figura 4). Si los usuarios consumirían agua de bidones debido a que desconfían de la calidad del agua de la red pública, se esperaría que existiese una relación entre esas variables. Los estratos socio económicos más altos consumen más bidones per cápita que los estratos socio económicos más bajos. En los 4 estratos socio económicos, el patrón común muestra que cuando el Ingecap aumenta, el consumo per cápita de bidones disminuye linealmente, como se esperaba. El $95.6 \%$ y $99.6 \%$ del estrato A y D respectivamente (tabla 7), indican que el consumo de bidones per cápita dependen de la calidad del agua de la red pública, evaluada a través del Ingecap. Tienen una correlación lineal perfecta, mientras que para los estratos B y C la correlación es considerable pero el coeficiente determinación es de baja significancia.

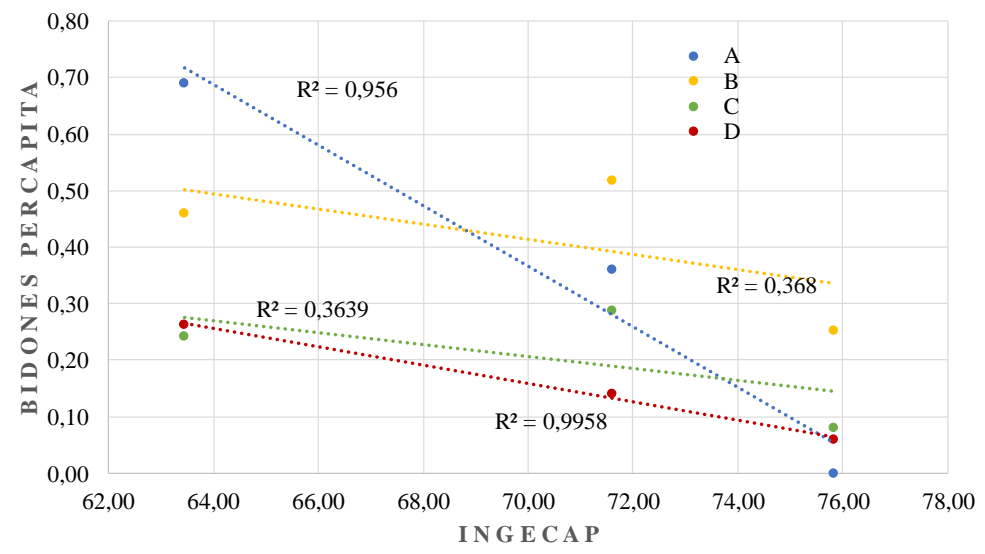

Figura 4: Ingecap y Bidones per cápita, por cada estrato y de las 11 ciudades (Lindao, 2018).

Tabla 7: Análisis estadístico Ingecap vs Bidones de agua, por estratos, de las 11 ciudades (Lindao, 2018).

\begin{tabular}{cccc} 
Estrato & $\mathrm{R}^{2}$ & $\mathrm{R}$ & Significancia $(\mathrm{R})$ \\
\hline $\mathrm{A}$ & 0.956 & 0.978 & Co. Perfecta \\
$\mathrm{B}$ & 0.368 & 0.607 & Considerable \\
$\mathrm{C}$ & 0.364 & 0.603 & Considerable \\
$\mathrm{D}$ & 0.996 & 0.998 & Co. Perfecta \\
\hline
\end{tabular}

Co. Perfecta: Correlación Perfect

\section{Conclusiones}

El objetivo del estudio fue determinar si existe relación entre el consumo de agua potable de la red pública y la calidad de esa agua. Para esto se debió correlacionar el consumo de agua embotellada como respuesta a la desconfianza en la calidad del agua pública. El indicador de calidad y de gestión del agua de la red pública es el Ingecap, es utilizado para cuantificar la gestión y calidad del agua potable de la red pública. 


\section{Unach}

La gestión del agua potable sería el principal factor que afecta la calidad del agua y consecuentemente del consumo de agua embotellada. La gestión se refiere a los análisis periódicos de la calidad del agua y a la provisión continua de la misma, por parte de las empresas públicas al sector residencial. Si esa gestión ha sido deficiente en cantidad y calidad, la reacción de los usuarios es de desconfianza e insatisfacción. La aparente insatisfacción o desconfianza de los usuarios sería la razón para que consuman agua embotellada, como lo reportó Doria (2006).

El Indicador de Gestión y de Calidad del agua potable, Ingecap, tiene correlaciones lineales muy fuertes y perfectas con el olor, color y sabor del agua de la red pública, lo que avalaría la confiabilidad del Ingecap como indicador. Requiere de la medición de 6 parámetros de calidad básicos del agua potable: color, turbiedad, $\mathrm{pH}$, cloro residual, coliformes totales y sólidos totales disueltos (STD) que no sería un limitante en países en desarrollo (OMS, 2002). El Ingecap podría ser un instrumento de control de respuesta rápida que arrojaría resultados confiables para tomar correctivos urgentes.

La calidad del agua de las ciudades pequeñas (Columbe, Cubijíes, Guamote, Chambo, Químiag y Guano) y medianas (Joya de los Sachas, Macas y Guaranda) es mejor que la de las ciudades grandes (Ventanas y Riobamba). El consumo de bidones es más alto en las ciudades grandes y en los estratos socio económicos más altos debido a que tienen menos limitaciones económicas que los estratos más bajos.

La percepción de tierra en el agua potable de la red pública por parte de los usuarios, estaría relacionada al uso de tanques de almacenamiento. Es evidente la influencia de la capacidad económica de los usuarios en los consumos de agua potable. El efecto del costo del agua de la red pública y otros factores concurrentes no han sido considerados en este estudio.

\section{Conflicto de Intereses}

Los autores declaramos que no existe ningún tipo de Conflicto de Interés.

\section{Referencias}

Ali Sajjadi, S., Alipour, V., Matlabi, M. \& Biglari, H. (2015). Consumer Perception and Preference of Drinking Water Sources. Electronic Physician, 7(1), 971-976. doi.org/10.14661/2015.971-976

Arellano, A., Bayas, A., Meneses, A. \& Castillo, T. (2018a). Los consumos y las dotaciones de agua potable en poblaciones ecuatorianas con menos de $150 \quad 000$ habitantes. NovaSinergia, 1(1), 23-32.

Arellano, A., González, J. \& Gavilanes, A. (2012). Método de Caracterización Urbanística y SocioEconómica para Poblaciones menores que 150.000 habitantes. Riobamba.

Barreno, K. (2015). Determinar la influencia de la situación socioeconómica, algunos factores meteorológicos y la calidad del agua, en el consumo de agua potable de la parroquia urbana del cantón La Joya de los Sachas perteneciente a la provincia de Orellana. Universidad Nacional de Chimborazo.

Cáceres, E. \& Rubio, V. (2015). Efectos de los factores Socioeconómicos, climatológicos $y$ de calidad del agua, que inciden en el consumo de agua potable, caso de estudio parroquias urbanas La Matriz y el Rosario del cantón Guano. Universidad Nacional de Chimborazo.

Carrillo, A. \& Quintero, H. (2013). Indicadores de cantidad y calidad del agua consumida en la ciudad de Riobamba. Universidad Nacional de Chimborazo.

Doria, M. (2006). Bottled water versus tap water: Understanding consumer's preferences. Journal of Water and Health, 4(2), 271276. doi.org/10.2166/wh.2006.008

Hernández Sampieri, R., Fernández Collado, C. \& Baptista Lucio, P. (2006). Metodología de la investigación (Cuarta). México: McGraw-Hill.

INEC. (2016). Medición de los indicadores ODS de Agua, Saneamiento e Higiene (ASH) en el Ecuador. Recuperado de: http://www.ecuadorencifras.gob.ec/docum entos/webinec/EMPLEO/2017/Indicadores ODS Agua, Saneamiento e Higiene/Presentacion_Agua_2017_05.pdf

INEC, I. E. de E. y C. (2010). Censo de poblacion $\mathrm{y}$ vivienda.

Instituto Ecuatoriano de Normalización INEN. (2011). Recuperado de: Norma-INENagua-potable_1108.pdf. Quito-Ecuador.

Lindao, V. (2018). Incidencia de la calidad de agua potable en el consumo diario residencial en poblaciones menores a 150.000 habitantes. Universidad Nacional de Chimborazo. Recuperado

de: 
http://dspace.unach.edu.ec/handle/51000/5

097

Montenegro, D. \& Tapia, Y. (2014). Indicadores de cantidad y calidad del agua consumida en la ciudad de Macas. Universidad Nacional de Chimborazo.

Morillo, P. \& Luna, M. (2013). Determinación de indacadores de cantidad y calidad del agua consumida en la ciudad de Ventanas. Universidad Nacional de Chimborazo.

Morote Seguido, Á. F. (2017). Factores que inciden en el consumo de agua doméstico. Estudio a partir de un análisis bibliométrico. Estudios Geográficos, $78(282)$, 257. doi.org/10.3989/estgeogr.201709

Noriega, D. (2015). Estudio del consumo de agua potable y de los principales factores que afectan la utilización del agua en el cantón Chambo, para optimizar el uso del recurso. Universidad Nacional de Chimborazo.

OMS. (2002). Guía para la vigilancia y control de la calidad del agua para consumo humano. Organización Mundial de La Salud, 353. doi.org/10.4172/2157-7145.S2-005

Patiño, J. \& Pino, F. (2014). Estudio del consumo de agua potable $y$ de los principales factores que afectan la utilización del agua en el cantón Guaranda, para optimizar el uso del recurso. Universidad Nacional de Chimborazo.

Rufener, S., Mäusezahl, D., Mosler, H. J. \& Weingartner, R. (2010). Quality of drinking-water at source and point-of consumption-Drinking cup as a high potential recontamination risk: A field study in Bolivia. Journal of Health, Population and Nutrition, 28(1), 34-41. doi.org/10.3329/jhpn.v28i1.4521

Sagñay, L. \& Carguachi, E. (2015). Análisis comparativo entre las características socioeconómicas, climatológicas y el gasto de agua potable de las parroquias Guamote y Columbe. Universidad Nacional de Chimborazo.

Torres, P., Cruz, C. \& Patiño, P. (2009). Índices de calidad de agua en fuentes superficiales utilizadas en la producción de agua para consumo humano. Una revisión crítica. Revista Ingenierías 8(15), 79-94. 